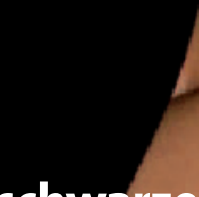

\section{Cave: Heller Hautkrebs auf schwarzem Tattoo!}

Tran ätowierungen gehen mit zahlreichen Komplikationen einher wie Entzündungen und der Übertragung von Infektionskrankheiten. Zwei Fälle von Plattenepithelkarzinomen, die sich direkt über Tätowierungen entwickelten, erweitern nun diese Nebenwirkungspalette. Beide Karzinome traten bei jüngeren Menschen auf - in einem Fall bei einem 35-jährigen Mann zehn Jahre nach Anbringen des Tattoos, in dem anderen Fall bei einer 30-jährigen Frau zehn Monate nach der Tätowierung. In beiden Fällen war das Plattenepithelkarzinom über schwarz tätowierten Bereichen lokalisiert, bei der Frau wurde im gleichen Bereich zusätzlich ein Keratoakanthom diagnostiziert. Über die Entstehungsmechanismen ist nichts bekannt. Risikofaktoren wie familiäre Be-

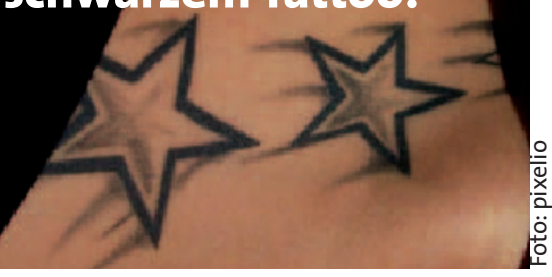

lastung, Sonnenexposition oder Immunsuppression lagen bei beiden Patienten nicht vor. Möglicherweise entwickelten sich die Karzinome als Folge einer chronischen Entzündungsreaktion gegen das schwarze Pigment, oder das Pigment war infektiös kontaminiert. Tatsächlich wurden in schwarzen Tattoo-Pigmenten humanpathogene Papillomaviren nachgewiesen.

$b k$

Pitarch G et al. J Am Acad Dermatol 2007; 56:1072-3

\title{
Heilpraktikerin außer Rand und Band
}

Eir ine Heilpraktikerin war bei einer wohlhabenden Familie gut im Geschäft. Sie bearbeitete Eltern und Sohn u. a. mit Iris-Diagnose, Mey-Immun-Präparaten, Eigenblutbehandlung, Akupunktur, Quaddelungen und Schröpfen. Nachdem sie dafür schon fast 120.000 DM kassiert hatte, trat der Familienvater auf die Bremse und blieb weitere Rechnungen über insgesamt $10.500 €$ schuldig. Die Zahlungsklage der Frau blieb ohne Erfolg. Werde ein Dienstvertrag schlecht erfüllt, berühre das normalerweise den Anspruch auf Vergütung nicht, stellte das Gericht fest. Wenn eine Leistung jedoch vollkom-

\section{Schön genäht!}

B ei einer 54-jährigen Frau aus England war im Mai 2005 nach einem Trauma eine Ellenbogengelenksprothese implantiert worden. Die Wunde heilte problemlos ab, allerdings entwickelte die Frau eine allergische entzündliche Reaktion auf das Nahtmaterial, die ohne Beschwerden auch noch ein Jahr nach der Operation anhielt. Unbeabsichtigt setzte sich der Chirurg selbst ein Denkmal für seine gleichmäßig exakte Nahttechnik. hsf men unbrauchbar sei, könne sich der Kunde darauf berufen, dass der Vertrag nicht erfüllt wurde. Die Heilpraktikerin habe ohne gesicherte Diagnose wahllos und in unerhörter Frequenz Therapiemaßnahmen durchgeführt und abgerechnet, denen kein plausibles Behandlungskonzept zugrundelag. Angesichts der nicht sehr gravierenden Befunde der Behandelten sei diese Art von „Heilbehandlung“ unbrauchbar oder sogar nachteilig für die Patienten. Diese hätten daher zu Recht die Zahlung des Honorars verweigert. gri

\section{Oberlandesgericht Koblenz, 12 U 1433/04}

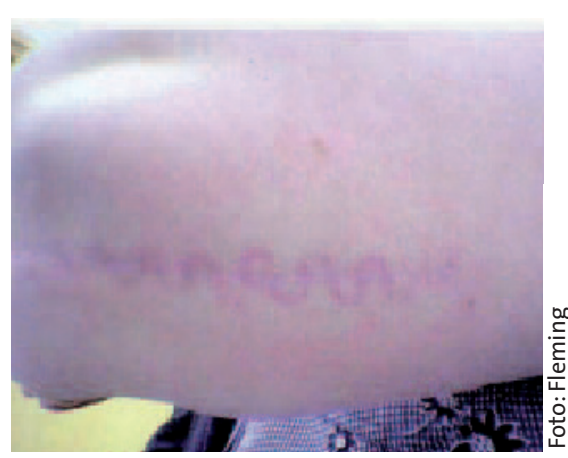

Fleming C et al. BMJ 2007; 334: 542

\section{Asthma wegatmen?}

$\sqrt{ }$ it einfachen Atemübungen können Asthmasymptome signifikant verringert werden. Das zeigt eine randomisierte Studie mit 85 Patienten. Die Studienteilnehmer, die zusätzlich zu ihrer normalen Therapie in fünf Sitzungen nach der Papworth-Methode instruiert wurden, hatten in den folgenden zwölf Monaten rund ein Drittel weniger Atemprobleme als konventionell behandelte Kontrollpatienten. Bei der PapworthMethode werden vor allem die Atmung durch die Nase und die „Bauchatmung“ gefördert.

van der Wouden JC et al. Thorax 2007; 62: 835

\section{Apfel neu - Allergie alt}

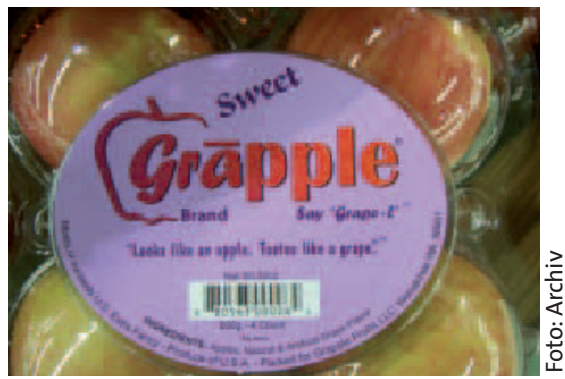

ieht aus wie ein Apfel, schmeckt wie eine Traube-mit diesem eingängigen Slogan bewirbt seit 2004 ein US-Lebensmittelkonzern ein neues Designobst: den Grapple. Jetzt berichtet ein deutsch-amerikanisches Forscherteam von den ersten beiden Fällen einer Nahrungsmittelallergie gegen Grapples. Im Pricktest reagierten beide Personen positiv sowohl auf Grapples als auch auf gewöhnliche Äpfel. Spezifisches IgE gegen Trauben konnte dagegen nicht nachgewiesen werden. Mit einem Blick auf den Produktionsprozess der Grapples überrascht das Ergebnis nicht: Die Frucht ist nicht etwa gezüchtet oder genetisch manipuliert, vielmehr werden herkömmliche Äpfel mit Wasser und dem künstlichen Traubenaroma Methylester behandelt. Echte Traubenproteine sind im Grapple also gar nicht enthalten. $\quad c g$

Otto HF et al. Int Arch Allergy Immunol 2007; 143: 211-15 